\title{
Neoadjuvant treatment with cisplatin and $S-1$ in elderly patients with oesophagogastric adenocarcinoma and locoregional disease: Two case reports and review of the literature
}

\author{
CASPAR FRANCK, ALI CANBAY, PETER MALFERTHEINER and MARINO VENERITO \\ Department of Gastroenterology, Hepatology and Infectious Diseases, Otto-von-Guericke \\ University Hospital, D-39120 Magdeburg, Germany
}

Received May 26, 2017; Accepted August 21, 2017

DOI: $10.3892 / \mathrm{mco} .2017 .1445$

\begin{abstract}
Perioperative platinum/fluoropyrimidine-based chemotherapy is the therapeutic standard for oesophagogastric cancer (OAC) patients with locoregional disease. The preoperative condition directly affects postoperative prognosis; thus, particularly for elderly patients, a perioperative regimen with a favourable side effect profile is highly desirable. In the palliative setting, the combination of cisplatin and $\mathrm{S}-1$ (Cis/S-1) was found to be as effective as cisplatin/5-fluorouracil, but with a more favourable side effect profile. However, no data on this combination have been reported in the perioperative setting in Caucasian patients. To the best of our knowledge, this is the first report on the treatment outcome of two elderly Caucasian OAC patients with locoregional disease receiving two neoadjuvant 4-week cycles of intravenous Cis/S-1. Both patients tolerated the doublet therapy well. No treatment delay or dose reduction was required. In both cases, preoperative staging revealed a clear response and complete surgical resection could be performed without any complications.
\end{abstract}

\section{Introduction}

Oesophagogastric adenocarcinoma (OAC), including adenocarcinoma of the gastro-oesophageal junction and stomach, is a major health concern, particularly in elderly patients (1). A recent study from the USA reported that approximately two-thirds of OACs were diagnosed at an advanced stage, with regional lymph node invasion or distant metastases (2). In cases with advanced $\mathrm{T}$ stage or regional lymph node involvement $\left(\mathrm{T} 3 / 4\right.$ or $\left.\mathrm{N}^{+}\right)$, without evidence of distant metastases,

Correspondence to: Dr Marino Venerito, Department of Gastroenterology, Hepatology and Infectious Diseases, Otto-von-Guericke University Hospital, Leipziger Street 44, D-39120 Magdeburg, Germany

E-mail: m.venerito@med.ovgu.de

Key words: cisplatin, elderly patients, oesophagogastric cancer, perioperative chemotherapy, $\mathrm{S}-1$ surgical resection with D2 lymph node dissection is indicated. The MAGIC trial in 2006 first demonstrated an improvement in 5-year survival from 23 to $36 \%$ in patients with resectable stage II and III OAC treated with six cycles of perioperative chemotherapy with 5-fluorouracil (5-FU), cisplatin and epirubicin (ECF regimen) compared with surgery alone, establishing perioperative cytostatic treatment as the new standard of care (3).

Since then, it has been demonstrated that epirubicin does not confer any additional benefit in terms of overall survival (OS) in patients undergoing preoperative chemotherapy for OAC (4). The European Society for Medical Oncology guidelines currently state that 'it may be reasonable to use any fluoropyrimidine-platinum doublet or triplet' (5). The treatment suggested herein, although not explicitly mentioned, includes cisplatin and S-1 (Cis/S-1).

The fluoropyrimidine S-1 contains tegafur (an inactive 5-FU prodrug) and the two enzyme inhibitors gimeracil and oteracil. These components improve the efficacy and safety of the cytostatic agent (6) namely tegafur, gimeracil, and oteracil. In Europe, S-1 has been approved in combination with cisplatin for the palliative treatment of advanced OAC. Furthermore, in Japan, S-1 monotherapy represents the standard of care in the adjuvant setting following OAC resection (7). In the FLAGS trial, including 1,053 patients with metastatic OAC, Cis/S-1 did not prolong OS, but exhibited a significantly improved safety profile compared with cisplatin/infusional 5-FU $(8,9)$. The same favourable side effect profile of Cis/S-1 may also be expected in the perioperative setting. Cis/S-1 has been proven to be feasible and effective for the perioperative therapy of Asian OAC patients, but experience with perioperative Cis/S-1 in Caucasian OAC patients has not been reported thus far $(10,11)$.

\section{Case reports}

Case 1. A 75-year-old male patient with an Eastern Cooperative Oncology Group (ECOG) performance status score of 1 presented at the Department of Gastroenterology, Hepatology and Infectious Diseases (Otto-von-Guericke University Hospital, Magdeburg, Germany) in July 2016 with appetite loss and postprandial pain in the upper abdomen. Ambulatory 

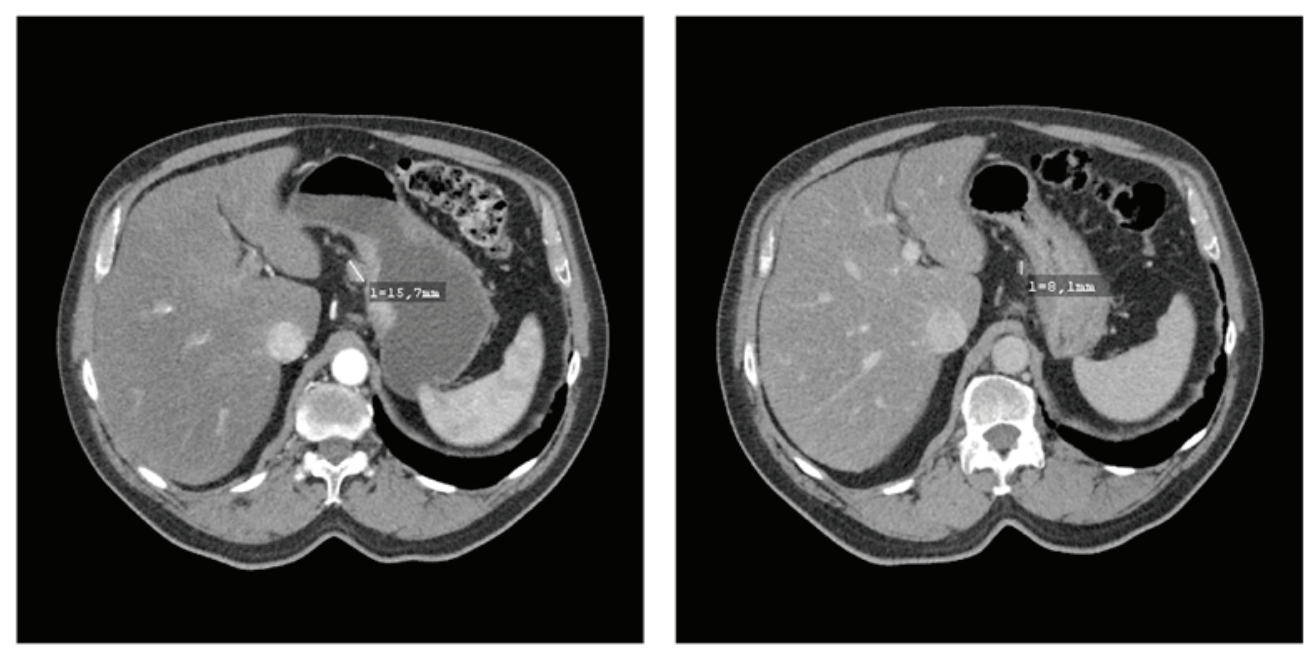

Figure 1. Case 1: Perigastric lymph node metastasis at the lesser curvature before (left) and after (right) neoadjuvant therapy on computed tomography examination.

oesophagogastroduodenoscopy (OGD) revealed a tumour at the gastro-oesophageal junction (Siewert type III). Repeated endoscopy with biopsy at our department confirmed the clinical suspicion. Histological examination revealed intestinal type adenocarcinoma according to the Laurén classification (12). The human epidermal growth factor receptor 2 (HER2) status was negative. There was no evidence of Helicobacter pylori infection. Staging computed tomography (CT) scan and endosonography revealed stage III disease (uT4uN3cM0) based on the 7th edition of the American Joint Committee on Cancer Staging Manual (2010) (13). Perioperative treatment with Cis/S-1 was initiated. Two preoperative 4-week cycles of intravenous cisplatin $75 \mathrm{mg} / \mathrm{m}^{2}$ on day 1 and oral S-1 $25 \mathrm{mg} / \mathrm{m}^{2}$ twice daily on days $1-21$ were administered. Apart from mild thrombocytopenia [grade I according to the Common Terminology Criteria for Adverse Events (CTCAE) v4.0 (14)] and a mild exanthema of the chest region (CTCAE v4.0 grade II), no further adverse events were observed. There was no treatment delay. The body weight remained stable during the entire course of the cytostatic treatment, and no deterioration of the ECOG score was observed. A preoperative CT scan revealed partial remission of the perigastric lymph node metastases and excluded distant metastases (Fig. 1). In early December 2016, radical gastrectomy with D2 lymph node dissection was performed. No complications occurred after surgery and the patient was discharged on the 11th postoperative day. The histopathological assessment of the resected tissue confirmed complete resection (R0) and proved partial regression (grade II according to Becker et al) with $20 \%$ residual tumour cells (15). After surgery, a further two cycles of cisplatin/S-1 were administered uneventfully. The last postoperative follow-up (June 2017) revealed no signs of tumour recurrence.

Case 2. A 71-year-old male patient in good general condition (ECOG performance status score 1) developed weakness and non-specific thoracic pain. The past medical history was remarkable for coronary heart disease, and the patient had already received two coronary stents. A recent percutaneous coronary angiography excluded significant stenoses. The laboratory tests revealed mild iron deficiency anaemia. The next diagnostic step was an OGD with biopsies, revealing adenocarcinoma of intestinal type (according to the Laurén classification) in the gastric antrum. HER2 status and Helicobacter pylori serology were negative. Staging CT scan and endosonography revealed locoregional disease without distant metastases (uT3uN1cM0). Perioperative cytostatic therapy with two 4-week cycles of Cis/S-1 was administered. The side effects included dysgeusia, appetite loss and mild recurring episodes of vomiting (CTCAE v4.0 grade II), which were successfully controlled with antiemetics. During the treatment, a weight loss of $5 \mathrm{~kg}$ (CTCAE v4.0 grade I) was observed. Therefore, dietary supplementation with high-calorie sip feed nutrition products was prescribed. In addition, a clinically non-relevant thrombocytopenia was observed (CTCAE v4.0 grade I). No treatment delay was deemed necessary. During neoadjuvant therapy, no deterioration of the ECOG score was observed. A preoperative CT scan revealed considerable shrinking of both the primary cancer and the regional lymph node metastases (partial response).

In December 2016, a minimally invasive gastrectomy with D2 lymphadenectomy was performed uneventfully. The postoperative course was uncomplicated and the patient was discharged on the 13th postoperative day. Histopathological examination revealed negative resection margins (R0). The histological tumour regression grade according to Becker et al was 3 (15). Between February and March 2017, two cycles of adjuvant cisplatin/S-1 were administered; however, a dose reduction was required (cisplatin $60 \mathrm{mg} / \mathrm{m}^{2}, \mathrm{~S}-120 \mathrm{mg} / \mathrm{m}^{2}$ twice daily) due to nausea (CTCAE v4.0 grade III). The last follow-up CT scan (July 2017) revealed no signs of tumour recurrence.

\section{Discussion}

We herein report the first two cases of Caucasian OAC patients receiving neoadjuvant $\mathrm{Cis} / \mathrm{S}-1$. Preoperative $\mathrm{Cis} / \mathrm{S}-1$ has been already investigated in Japanese OAC patients $(10,11)$. However, the efficacy and side effect profile of $\mathrm{S}-1$ is different between Asian and Caucasian subjects due to the differences in metabolism (16). Thus, the results of those studies may 
not be transferable to Caucasian patients. In both reported cases, no serious adverse events (CTCAE v4.0 grade III/IV) were observed preoperatively, and no therapy delay or dose reduction was required. Both patients were in a good preoperative condition and the staging revealed considerable tumour shrinkage. Surgery was performed without any complications, and tumour resection with negative margins (R0) was histologically confirmed in both cases.

The only chance for cure of non-metastatic OAC is complete resection. Perioperative chemotherapy improves $\mathrm{OS}$ in OAC patients with locoregional disease $(3,5,17,18)$. However, as gastrectomy and particularly oesophagectomy are high-risk procedures, the patient's preoperative general condition is crucial for the success of the interdisciplinary therapy approach. This is relevant, as OAC mostly occurs in elderly patients, and chronological age is a marker for increased physical frailty.

Whether elderly OAC patients with locoregional disease should receive perioperative triplet or a doublet chemotherapy has been investigated in recent trials. In a subgroup analysis of the FLOT65+ trial [5-FU, leucovorin and oxaliplatin with (FLOT) or without (FLO) docetaxel], the FLOT group exhibited increased chemotherapy-related toxicity and deterioration of quality of life global health status scores during the first 8 weeks of treatment compared with the FLO group (19). Another randomized study, which compared the triplet epirubicin, cisplatin and capecitabine (ECX) with the doublet CX (i.e., without epirubicin), yielded comparable efficacy results for both regimens (20). It should be noted that, in that trial, no patients in the CX and $12 \%$ of the patients in the ECX arm discontinued treatment due to toxicity. In summary, due to its inferior safety profile and potential deterioration of the preoperative general condition, neoadjuvant triplet chemotherapy should be discouraged in elderly OAC patients.

Although a platinum/fluoropyrimidine doublet regimen represents the standard of care in the perioperative therapy of OAC patients with locoregional disease, it is debatable whether perioperative regimens should be cisplatin- or oxaliplatin-based. Furthermore, no studies comparing perioperative $\mathrm{Cis} / \mathrm{S}-1$ and FLO are available. However, cisplatin and oxaliplatin have been compared as first-line treatment of advanced oesophagogastric cancer (21-23). In a German study comparing FLO to infusional 5-FU plus cisplatin (FLP regimen), oxaliplatin was safer with respect to haematological and non-haematological toxicity (i.e. nausea, vomiting and renal toxicity), but was associated with a significantly higher rate of peripheral polyneuropathy (22). In a recent phase III study comparing S-1/oxaliplatin (SOX regimen) and Cis/S-1 in Japanese OAC patients, similar results were obtained with respect to the safety issues, whereas no significant difference in terms of progression-free survival (PFS) and OS were observed between the two regimens $(24,25)$. Haematological toxicity is reversible, nausea and vomiting are preventable, whereas renal toxicity can be monitored. On the contrary, oxaliplatin-induced polyneuropathy is frequently irreversible and may even worsen after withdrawal of the drug, consistently compromising the quality of life in OAC survivors (26). In our experience, oxaliplatin-induced peripheral polyneuropathy occurs early in the adjuvant (postoperative) phase of perioperative treatment, leading to withdrawal of the drug. In view of the long-term neurotoxic sequelae of oxaliplatin and the lack of effective treatment options for this side effect, cisplatin-based chemotherapy may be preferred in the perioperative setting.

The fluoropyrimidine S-1 has shown favourable safety and efficacy data as palliative treatment of OAC. The FLAGS trial demonstrated significant improvements of tolerability due to the treatment with Cis/S-1 compared with Cis/5-FU, whereas OS and PFS did not differ significantly $(8,27)$. At least one treatment-related serious adverse event (CTCAE grade $>$ II) was observed in $29.7 \%$ in the Cis/5-FU arm compared with $20.5 \%$ in the $\mathrm{Cis} / \mathrm{S}-1 \mathrm{arm}$. Treatment-related deaths were also significantly more common in the Cis/5-FU group (4.9 vs. $2.5 \%$ ). In addition to the favourable side effect profile, other positive aspects of the Cis/S1 therapy should be highlighted. Due to the 4-week cycles, only one cisplatin infusion per month is necessary. This may result in i) improved quality of life, ii) reduced frequency of visits to the oncology department, and iii) reduced disease perception. The gained time may be invested in physical exercise and other coping strategies for a further improvement of the outcome, provided the patient's compliance is ensured.

In our experience, neoadjuvant and possibly perioperative Cis/S-1 represents a feasible, effective and well-tolerated treatment option for elderly Caucasian OAC patients with locoregional disease.

All procedures followed were in accordance with the ethical standards of the responsible committee on human experimentation (institutional and national) and with the Helsinki Declaration of 1964 and later versions. Informed consent or substitute for it was obtained from both patients.

\section{Acknowledgements}

Dr Marino Venerito received honoraria from Merck Serono and Bayer Vital and is a member of the of advisory boards of Amgen, Lilly and Nordic Pharma.

\section{References}

1. Robert Koch Institute and the Association of Population-based Cancer Registries in Germany: Cancer in Germany 2009/2010, 2014.

2. Jin H, Pinheiro PS, Callahan KE and Altekruse SF: Examining the gastric cancer survival gap between Asians and whites in the United States. Gastric Cancer 20: 1-10, 2016.

3. Cunningham D, Allum WH, Stenning SP, Thompson JN, Van de Velde CJ, Nicolson M, Scarffe JH, Lofts FJ, Falk SJ, Iveson TJ, et al; MAGIC Trial Participants: Perioperative chemotherapy versus surgery alone for resectable gastroesophageal cancer. $\mathrm{N}$ Engl J Med 355: 11-20, 2006.

4. Alderson D, Langley RE, Nankivell MG, Blazeby JM, Griffi M, Crellin A and Cunningham D: Neoadjuvant chemotherapy for resectable oesophageal and junctional adenocarcinoma: results from the UK Medical Research Council randomised OEO5 trial (ISRCTN 01852072). J Clin Oncol 33: suppl; abstr 4002, 2015.

5. Smyth EC, Verheij M, Allum W, Cunningham D, Cervantes A and Arnold D; ESMO Guidelines Committee: Gastric cancer: ESMO Clinical Practice Guidelines for diagnosis, treatment and follow-up. Ann Oncol 27 (suppl 5): v38-v49, 2016.

6. Kobayakawa M and Kojima Y: Tegafur/gimeracil/oteracil (S-1) approved for the treatment of advanced gastric cancer in adults when given in combination with cisplatin: A review comparing it with other fluoropyrimidine-based therapies. Onco Targets Ther 4: 193-201, 2011.

7. Shen L, Shan YS, Hu HM, Price TJ, Sirohi B, Yeh KH, Yang YH, Sano T, Yang HK, Zhang X, et al: Management of gastric cancer in Asia: Resource-stratified guidelines. Lancet Oncol 14: e535-e547, 2013. 
8. Ajani JA, Rodriguez W, Bodoky G, Moiseyenko V, Lichinitser M, Gorbunova V, Vynnychenko I, Garin A, Lang I and Falcon S: Multicenter phase III comparison of cisplatin/S-1 with cisplatin/ infusional fluorouracil in advanced gastric or gastroesophageal adenocarcinoma study: The FLAGS trial. J Clin Oncol 28: $1547-1553,2010$.

9. Mahlberg R, Lorenzen S, Thuss-Patience P, Heinemann V, Pfeiffer P and Möhler M: New Perspectives in the Treatment of Advanced Gastric Cancer: S-1 as a Novel Oral 5-FU Therapy in Combination with Cisplatin. Chemotherapy 62: 62-70, 2017.

10. Kunisaki C, Makino H, Kimura J, Takagawa R, Kanazawa A, Ota M, Kosaka T, Ono HA, Akiyama $\mathrm{H}$ and Endo I: Impact of S-1 plus cisplatin neoadjuvant chemotherapy on scirrhous gastric cancer. Oncology 88: 281-288, 2015.

11. Okabe H, Hata H, Ueda S, Zaima M, Tokuka A, Yoshimura T, Ota S, Kinjo Y, Yoshimura K and Sakai Y; Kyoto University Surgical Oncology Group (KUSOG): A phase II study of neoadjuvant chemotherapy with S-1 and cisplatin for stage III gastric cancer: KUGC03. J Surg Oncol 113: 36-41, 2016.

12. Lauren P: The two histological main types of gastric carcinoma: Diffuse and so-called intestinal-type carcinoma. An attempt at a histo-clinical classification. Acta Pathol Microbiol Scand 64: 31-49, 1965.

13. Edge SB, Byrd DR, Compton CC, Fritz AG, Greene FL and Trotti A (eds): AJCC Cancer Staging Manual. 7th edition. Springer, NY, 2010.

14. Common Terminology Criteria for Adverse Events (CTCAE) Version 4.0, published May, 2009. https://evs.nci.nih.gov/ftpl/ CTCAE/CTCAE_4.03_2010-06-14_QuickReference_5x7.pdf. Accessed April $2 \overline{3}, 201 \overline{7}$.

15. Becker K, Mueller JD, Schulmacher C, Ott K, Fink U, Busch R, Böttcher K, Siewert JR and Höfler H: Histomorphology and grading of regression in gastric carcinoma treated with neoadjuvant chemotherapy. Cancer 98: 1521-1530, 2003.

16. Chuah B, Goh BC, Lee SC, Soong R, Lau F, Mulay M, Dinolfo M, Lim SE, Soo R, Furuie T, et al: Comparison of the pharmacokinetics and pharmacodynamics of $\mathrm{S}-1$ between Caucasian and East Asian patients. Cancer Sci 102: 478-483, 2011.

17. Ajani JA, D'Amico TA, Almhanna K, Bentrem DJ, Chao J, Das P, Denlinger CS, Fanta P, Farjah F, Fuchs CS, et al: Gastric Cancer, Version 3.2016, NCCN Clinical Practice Guidelines in Oncology. J Natl Compr Canc Netw 14: 1286-1312, 2016.

18. Moehler M, Al-Batran SE, Andus T, Anthuber M, Arends J, Arnold D, Aust D, Baier P, Baretton G, Bernhardt J, et al; AWMF: German S3-guideline 'Diagnosis and treatment of esophagogastric cancer'. Z Gastroenterol 49: 461-531, 2011.

19. Lorenzen S, Pauligk C, Homann N, Schmalenberg H, Jäger E and Al-Batran S-E: Feasibility of perioperative chemotherapy with infusional 5-FU, leucovorin, and oxaliplatin with (FLOT) or without (FLO) docetaxel in elderly patients with locally advanced esophagogastric cancer. Br J Cancer 108: 519-526, 2013.

20. Yun J, Lee J, Park SH, Park JO, Park YS, Lim HY and Kang WK: A randomised phase II study of combination chemotherapy with epirubicin, cisplatin and capecitabine (ECX) or cisplatin and capecitabine (CX) in advanced gastric cancer. Eur J Cancer 46: 885-891, 2010.
21. Cunningham D, Starling N, Rao S, Iveson T, Nicolson $M$ Coxon F, Middleton G, Daniel F, Oates J and Norman AR; Upper Gastrointestinal Clinical Studies Group of the National Cancer Research Institute of the United Kingdom: Capecitabine and oxaliplatin for advanced esophagogastric cancer. N Engl J Med 358: 36-46, 2008.

22. Al-Batran S-E, Hartmann JT, Probst S, Schmalenberg H, Hollerbach S, Hofheinz R, Rethwisch V, Seipelt G, Homann N, Wilhelm G, et al; Arbeitsgemeinschaft Internistische Onkologie: Phase III trial in metastatic gastroesophageal adenocarcinoma with fluorouracil, leucovorin plus either oxaliplatin or cisplatin: A study of the Arbeitsgemeinschaft Internistische Onkologie. J Clin Oncol 26: 1435-1442, 2008

23. Yamada Y, Higuchi K, Nishikawa K, Gotoh M, Fuse N, Sugimoto N, Nishina T, Amagai K, Chin K, Niwa Y, et al: Phase III study comparing oxaliplatin plus S-1 with cisplatin plus S-1 in chemotherapy-naïve patients with advanced gastric cancer. Ann Oncol 26: 141-148, 2015.

24. Bando H, Yamada Y, Tanabe S, Nishikawa K, Gotoh M, Sugimoto N, Nishina T, Amagai K, Chin K, Niwa Y, et al: Efficacy and safety of S-1 and oxaliplatin combination therapy in elderly patients with advanced gastric cancer. Gastric Cancer 19: 919-926, 2016.

25. Nishikawa K, Yamada Y, Ishido K, et al: Impact of progression type on overall survival in patients with advanced gastric cancer based on randomized phase III study of S-1 plus oxaliplatin versus S-1 plus cisplatin. Gastric Cancer, 2016.

26. Burakgazi AZ, Messersmith W, Vaidya D, Hauer P, Hoke A and Polydefkis M: Longitudinal assessment of oxaliplatin-induced neuropathy. Neurology 77: 980-986, 2011

27. Ajani JA, Buyse M, Lichinitser M, Gorbunova V, Bodoky G, Douillard JY, Cascinu S, Heinemann V, Zaucha R, Carrato A, et al: Combination of cisplatin/S-1 in the treatment of patients with advanced gastric or gastroesophageal adenocarcinoma: Results of noninferiority and safety analyses compared with cisplatin/5-fluorouracil in the First-Line Advanced Gastric Cancer Study. Eur J Cancer 49: 3616-3624, 2013. 